\title{
Téoros
}

Revue de recherche en tourisme

\section{Rome, les pèlerinages, les jubilés}

\section{Pierre Calimé}

Volume 16, numéro 2, été 1997

Tourisme et religion

URI : https://id.erudit.org/iderudit/1074573ar

DOI : https://doi.org/10.7202/1074573ar

Aller au sommaire du numéro

Éditeur(s)

Université du Québec à Montréal

ISSN

0712-8657 (imprimé)

1923-2705 (numérique)

Découvrir la revue

Citer cet article

Calimé, P. (1997). Rome, les pèlerinages, les jubilés. Téoros, 16(2), 10-13.

https://doi.org/10.7202/1074573ar d'utilisation que vous pouvez consulter en ligne.

https://apropos.erudit.org/fr/usagers/politique-dutilisation/ 


\title{
$+\ldots . . .$. \\ ROME, \\ LeS PÈleRINAGeS, LeS JUBILÉS
}

\author{
Pierre Calimé, consulteur au Conseil pontifical \\ pour la Pastorale des Migrants et Itinérants
}

Les premiers lieux de pèlerinages chrétiens sont, bien sûr, les lieux saints du pays du Christ et * ce grand trou dans la terre qu'y fit la croix lorsqu'elle fut plantée et qui attire tout à elle, le centre et l'ombilic de la terre $x^{\prime}$.

La communauté chrétienne de Jérusalem conserve la mémoire des lieux de la Passion et de la Résurrection; elle les visite pour * faire mémoire $\%$. Du moins, tant que cela est possible : quand Elia Capitolina remplace la Sainte Citế après la seconde révolte juive (132-135), le Capitole recouvre le Golgotha et le Saint Sépulcre. Pourtant, Justin de Naplouse, en 150 , relate que de grandes foules se rendent à Bethléem pour vénérer la grotte de la Nativité et Méliton de Sardes, vers 175 , écrit à Onésime qu'il a visité ce territoire $\alpha$ où s'accroche le début de notre prédication et où s'accomplit ce qui est écrit dans la Bible $s$.

Le pèlerinage d'Hélène, mềre de l'empereur Constantin en 326, l'Invention de la Vraie Croix et, en 335 , la consécration de l'Anastasis, l'église de la Résurrection, ouvrent une ère de pèlerinage.

Le premier guide de pèlerinage en terre sainte, tinerarium Burdigada Jerusalem usque, sera écrit par un bordelais dès 333. Éthérie, * religieuse s espagnole de grande famille, laissera un récit de voyage (393-396) du plus haut interêt sur les pratiques de l'Église de Jérusalem en matière de célébration et d'accueil des pèlerins.
Prise de Jérusalem par les musulmans, en $637: 1^{\prime}$ avenir du pèlerinage est à Rome et aux hauts lieux européens: le MontSaint-Michel, et, surtout Saint-Jacquesde-Compostelle (fin IXe siècle).

\section{NAISSANCE DU PÈLERINAGE AD LIMINA APOSTOLORUM}

Rome, pour les chrétiens, plus que capitale de l'empire, est la Ville du martyr de Pierre (64-67) et de Paul (65-67). Selon la tradition, la mémoire de Pierre est honorée sur une tombe de pauvre, dans le cimetière situé entre le cirque de Néron et la colline vaticane ; celle de Paul sur la route d'Ostie. Les fouilles sous la basilique Saint-Pierre, décidées par Pie XII en deux campagnes (1939-1949 et 1953-1958), montrent qu'une tombe semble avoir toujours été respectée et honorée dès l'origine. Une des tuiles qui la couvre porte le sceau de Vespasien (6979). On sait par la lettre du prêtre Caiüs (vers 200) qu'un trophée a été construit (vers 160) sur la memoria de l'Apôtre Pierre. De Constantin au Bernin, tous les édifices et ornements seront centrés sur cette memoria, cceur de la Rome chrétienne.
Avec la paix donnée par Constantin (312$327)$, commence pour la communauté chrétienne, urbaine, un véritable : établissement $\%$. Une couronne de grandes basiliques va s'édifier à la périphếrie de la Ville, sur les lieux les plus porteurs de mémoire : Saint-Pierre, au Vatican ; Saint-Jean-de-Latran ; Saint-Laurent, à l'est ; Sainte-Agnès, au nord-est. SaintPaul devra se contenter au début d'une chapelle sur l'Ostiense.

On commence à honorer les martyrs. Les évêques de Rome comprennent vite qu'il leur faut structurer la vie religieuse de leur communauté grandissante. Dans la première moitié du IVe siècle, Sylvestre, Marc, Jules, Libère vont unir leurs ressources pour bâtir les premières églises urbaines, centres de prédication et de culte, sur les lieux où se garde la mémoire des martyrs: Chrysogone, Cécile, Sabine, Priscille, Balbine, Étienne, Jean et Paul, Clément, Pierre aux liens pour ne citer que ceux-ci. Ces églises * titres * sont donc liées au souvenir des martyrs et drainent les chrétiens de la Ville et d'ailleurs.

Le pape Damase (366-386) organise le culte des saints qui « mobilise les dévotions de la piété locale $*$ et tente * d'en modérer le zèle $\%$ (Pietri : 1976, 83). Les accès des catacombes, qui servent de moins en moins de cimetières et de plus en plus de lieux de pèlerinage, sont aménagés. Cette conquête de l'espace se conjugue avee une maîtrise du temps : le calendrier des célébrations se constitue. Les dates de Noẻl et de Pâques sont fixées dès le IV" siècle, ainsi que l'Avent et le Carême, repoussant la mémoire des saints hors de ces temps privilégies. 
À cette dévotion croissante va s'ajouter le prestige de l'Église de Rome et de son Évêque. On y viendra pour vénérer les martyrs, emporter des reliques, précieuses pour la fondation de monastères ou le renom d'une cathédrale, et pour confirmer la foi près du * siège de Pierre \%. Si Pierre et Paul sont honorés ensemble le 29 juin, dès 258 la fête de la chaire de Saint-Pierre à Rome est instituée le 22 février. On viendra donc ad limina apostolorum et, de plus en plus, quand le pouvoir spirituel du pape grandira, pour confirmer sa foi, recevoir des autorisations, solliciter sa réconciliation, être reconnu dans ses fonctions.

Princes et évêques viennent trouver près de Pierre leur légitimité, demander des reliques, faire pénitence et quêter la réconciliation, solliciter un encouragement pour la fondation de monastère, demander justice. Le flot de pèlerins ne cesse de croître.

Dès 400 on dresse des « itinéraires \%, sortes de catalogues ordonnés des monuments et des lieux remarquables de la Ville. La Notitia, transcrite à la suite des Chronographes de 334 est le premier almanach qui énumère les fêtes, la naissance des saints à la vie glorieuse - le natalis - précisant les lieux de leur sếpulture; les fêtes consulaires, les dates de Pâques, le catalogue des évêques depuis saint Pierre et, pour compléter le tout, la liste des préfets de la Ville et des fêtes païennes. C'est le premier " guide du parfait pèlerin $\%$. D'autres suivront : les « Itinéraires $*$ du prêtre Jean $\left(V^{*}\right)$, de Strasbourg (époque de Honorius I ${ }^{\mathrm{e}}$, 625630), d'Einsiedeln (époque de Charlemagne), Mirabilia Urbis Romæ, époque du pape Hadrien $\left(\mathrm{VIII}^{2}-\mathrm{IX}^{*}\right)$.

Les monastères ont le devoir d'accueillir les pèlerins. Les * guides » (mostratores) apparaissent comme un corps de métier, ainsi que les préposés qui expliquent aux pèlerins ce qu'ils voient, racontent la vie des martyrs ; les chanteurs et les bateleurs débitent les louanges du saint dans de longues cantilènes qui, à leur tour, donnent naissance aux cantiques de pèlerinages. On conserve un petruslied que les germaniques chantaient à Saint-Pierre au IX siècle.

On voit déjà naître les « souvenirs de pèlerinage s: brandea et palliola, morceaux d'étoffe qui ont touché la tombe vénérée, mais aussi des objets de tous genres, des ampoules de l'huile qui éclaire la tombe du saint... * Cette discipline et cette organisation, à l'origine, sont faites pour convertir les habitants de la Ville, mais elles préparent et appellent le pèlerinage * (Chelini et Branthomme, $1982: 85$ ).

Tout l'arsenal du pèlerinage populaire existe déjà.

Du VIII ${ }^{e}$ au $\mathrm{XIV}^{e}$ siècle, le pèlerinage continuera à se développer de manière diversifiée. Rome demeurera longtemps * le réservoir universel des reliques * et le centre de la catholicité. Le développement des sanctuaires en Europe modifiera la pratique du pèlerinage à Rome. La méfiance croissante des évêques et des docteurs contribuera également à freiner le pèlerinage populaire.

Au XIVe et au XVI $\mathrm{XV}^{\circ}$ siècles, l'idée du pèlerinage-pénitence évolue et le courant, déjà existant de méfiance envers le pèlerinage va se développer au point d'en ruiner sensiblement la pratique. \& Rigueur et prudence de la part des prélats ; sarcasmes et censures de la part des gens de lettres et docteurs w vont ouvrir une contestation radicale du pèlerinage au début de l'époque moderne, même si le XVII ${ }^{t}$ siècle sera marqué par un puissant renouveau (Chelini et Branthomme, 1982 : 231). Le Siècle des lumières confirmera le déclin du pèlerinage.

C'est étrangement au moment des difficultés entre le pape et l'Italie, autour des années 1870 , que va s'amorcer le renouveau du pèlerinage romain, à partir de la Belgique et de la France surtout. Le rayonnement de la papauté, de Léon XIII et de Pie X principalement, va renforcer le mouvement des pèlerinages, un temps arrêté par la guerre de 1914-1918 et, à nouveau entre 1939 et 1945 . L'ampleur des flux sera telle que Paul VI décidera la construction de la salle d'audience (architecte Nervi) de plus de 6000 places. Jean Paul II double fréquemment les audiences en raison de l'affluence.

\section{LES JUBILÉS}

En 1299 , le bruit court que le pape célébrerait une année jubilaire : les pèlerins pourraient recevoir l'indulgence plénière. Boniface VIII n'avait rien pensé de tel, mais, devant l'affluence des foules, il consentit le 22 février 1300 (fête de la Chaire de Saint-Pierre) à leur donner raison. Ce fut le premier jubilé. $\mathrm{Y}$ eut-il deux millions de pèlerins ? Villani, un florentin, affirme qu'il n'y eut jamais moins de 200000 pèlerins présents dans la Ville. Il faut probablement revoir ce chiffre à la baisse, comme celui des prévisions pour l'année 2000 !

La bulle d'indiction prévoyait un jubilé tous les siècles. Clément VI (1342-1352) réduisit de moitié l'intervalle, En 1390, le pape en avança la célébration de dix ans. En 1423, Martin V célébra les 33 ans écoulés depuis le jubilé précédant (33 ans : la vie du Christ) et en 1450 , Nicolas V instaura le rythme de 25 ans qui, selon les aléas de l'histoire, fut maintenu.

Jean Paul II a accéléré le rythme des années saintes en instituant, en 1983, l'année sainte de la Rédemption et, en 1987-1988, l'année sainte mariale. Le pape lui-même mentionne dans sa lettre Tertio Millennio Adveniente $\left(\mathrm{n}^{\circ} 27\right)$ qui décide de la célébration du jubilé de l'an 2000 : « L'année mariale a été comme une anticipation du jubilé, elle contenait déjà bien des éléments qui devront être pleinement exprimés en l'an $2000 \mathrm{~m}$.

Il faut encore noter que dans sa première encyclique, Redemptor Hominis (1979), Jean Paul II écrivait déjà : * Pour l'Église... cette année-là (l'an 2000) sera une année de grand jubilé $\%$. Sa décision de préparer le jubilé par trois années de méditation est inscrite dès le début dans le programme de son pontificat.

\section{LE JUBILÉ DE L'AN 2000}

Le mot vient du mot hébreux jobel, corne de bélier, la fête du jubilé étant annoncée par des sonneries de cors faits avec ces comes.

Dans l'Ancien Testament, tous les sept ans avait lieu un année sabbatique, et toutes les sept fois sept ans une année jubilaire. Années de repos de la terre, de remise des dettes, de libération des esclaves, de mise à plat de l'ordre social. * Chacun rentrera dans son patrimoine, chacun retournera dans son clan * (Lev 21,10 ). 
La lettre d'indiction du $28^{\circ}$ Jubilé de l'histoire de l'Église catholique, Tertio Millennio Adveniente (vers l'an 2000), est datée du 10 Novembre 1994 et structure la préparation et la célébration de ce Grand jubilé de l'an 2000 de maniềre nouvelle.

Au lieu d"un seul Cardinal-Président et d'une vaste assemblée sans signification particulière, le pape met en place deux instruments : un Comité de présidence de cing cardinaux et un Comité central, limité en nombre (32), dont les membres ne sont pas nommés au titre de leur appartenance à un Dicastere de la Curie romaine, mais en raison d'une mission précise en vue du jubilé.

Le Conseil des cardinaux comprend l'Archiprêtre de la Basilique Saint-Pierre, Cardinal Noe ; le vicaire de Rome, Cardinal Ruini (les accueillants des pelerins) : les cardinaux Cassidy (président du Conseil pour l'unité des chrétiens), Arinze (président du Conseil pour le dialogue inter-religieux) et Etchegaray (président du Conseil Justice et Paix et maître d'ouvre de l'ensemble). Le cadre est donné de ce que doit être la célébration du jubilé : la recherche du dialogue avec toutes les religions, la recherche de l'unité entre les chrétiens, la recherche de la justice et de la paix.

Le Comité central, doté d'un Secrétaire général, est composé d'évêques responsables des diverses commissions : pastorale, liturgique, théologique, dialogue inter-religieux, ecuménique, pour le martyrologe, artistico-culturelle, sociale. Siègent en outre les Secrétaires généraux des Conférences épiscopales continentales, les représentants des Comités de Rome et de Jérusalem et les présidents des Comités techniques et des médias. Deux femmes sont membres du Comité central avec un plein droit de parole sur tous les sujets, au titre de laiques ayant des responsabilités dans le monde et dans l'Église.

Une autre nouveauté : les jubilés étaient toujours célébrés a Rome et, éventuellement, l'année suivante dans les églises particulières. La lettre apostolique indique clairement : * La célébration même du Grand jubile aura lieu simultanément en terre sainte, à Rome et dans les églises locales du monde entier $\gg\left(\right.$ TMA $\left.^{\circ} 55\right)$. Cette norme ne vise pas d'abord à freiner le pèlerinage à Rome, mais à favoriser la célébration, dans les églises locales, des trois années consacrées à la méditation sur le Christ (1996-1997), a l'Esprit Saint (1997-1998), à Dieu le Père (1998-1999) et la glorification de la Trinité, mystère central de la foi chrétienne (1999-2000).

Trois années de préparation confiées aux diocèses et aux paroisses du monde entier, pour une célébration permettant à tous, y compris aux plus pauvres, de bénéficier des fruits spirituels de tout jubilé.

La note \& sociale est accentuée par le pape : Il existait une conviction commune : à Dieu seul, en tant que Créateur, appartient le 'dominium altum ', c'est-àdire la seigneurie sur toute la création, en particulier sur lat terre... C'est pourquoi les richesses de la création doivent être considérés comme un bien commun de l'humanité entière $\%$ (TMA ${ }^{\circ} 13$ ). Et les conclusions sont tirées : le jubilé doit prendre en compte * la sauvegarde de la dignité et des droits de la personne dans le cadre d'un juste rapport entre travail et capital et la promotion de la paix * (TMA $\mathrm{n}^{\circ} 22$ ) et w les chrétiens devront se faire la voix de tous les pauvres du monde, proposant que le jubilé soit un moment favorable pour penser à une réduction importante sinon à un effacement total de la dette internationale qui pèse sur le destin de nombreuses nations $*$ (TMA, $\mathrm{n}^{\circ} 51$ ).

On est très loin d"une vision étroitement touristique ou strictement piétiste de la célébration du jubilé.

\section{JUBILÉ : TOURISME OU PÈLERINAGE ?}

Quel que soit le contenu de la célébration du jubilé, son annonce a déclenché à Rome et en Italie une furie d'estimations et de projets pharaoniques. On a pu entendre le chiffre de 60 millions de pèlerins-visiteurs. Le nombre officiel de visiteurs des musées Vatican en 1995 était de trois millions!

Le Comité central de l'année sainte, sous l'autorité du Conseil des cardinaux, s'occupe en priorité et quasi uniquement des aspects spirituels de l'événement.

Une commission mixte Église-État a été constituée pour étudier les problèmes logistiques communs dans le respect des compétences réciproques.

Il est du ressort de l'Église de trouver des lieux de célébrations capables d'accueillir des assemblées de plusieurs centaines de milliers de personnes. À titre de référence, la béatification de Sr Bakhita

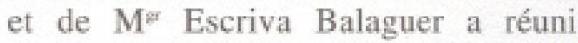
350000 personnes. Cela veut dire la place Saint-Pierre, la place Pie XII et la totalité de la via della Conciliazione jusqu'au Tibre... Difficile d'assurer la communion dans une telle dispersion.

Outre la délicate question de l'accueil pastoral des groupes et des individus dans les grandes basiliques, les problèmes logistiques, du strict point de vue touristique, sont de tous ordres : logement des personnes valides et des malades; repas ; protection des personnes, des biens, de la santé ; problèmes de circulation et de stationnement ; information sur place et à partir des frontières pour aiguiller les arrivants vers des zones où l'accueil est possible ; régulation préalable des flux de pèlerins organisés et, plus encore, des pèlerins-visiteurs isolés. Quand on sait qu'à Lourdes les quatre cinquièmes des cinq millions de visiteurs sont des familles, on voit que la vraie question de l'accueil n'est pas celle des groupes constitués.

Le spectacle de la Ville les jours d'audience pontificale en période de pointe touristique laisse entrevoir les difficultes d'écoulement de la circulation si les foules annoncées sont au rendez-vous romain. Des projets ont été avancés : * délocalisation * maximale en logeant les pèlerins le plus loin possible de Rome (jusqu’à Naples, Bologne, Florence...) et en les transportant par train pour un séjour de moins de 24 heures.

De grands travaux ont été envisagés pour faciliter la circulation déjà difficile : passage souterrain devant le Château SaintAnge ou son $\alpha$ contournement $w$, stationnement pour les autocars sous la colline du Janicule ; création d'une ligne de métro joignant la gare centrale à SaintPierre. Il est difficile de savoir où en sont exactement ces projets.

Le Touring Club italien a rendu public quelques chiffres intéressants : on ne peut accueillir plus de 18 millions de personnes en un an dans les structures hôtelières 


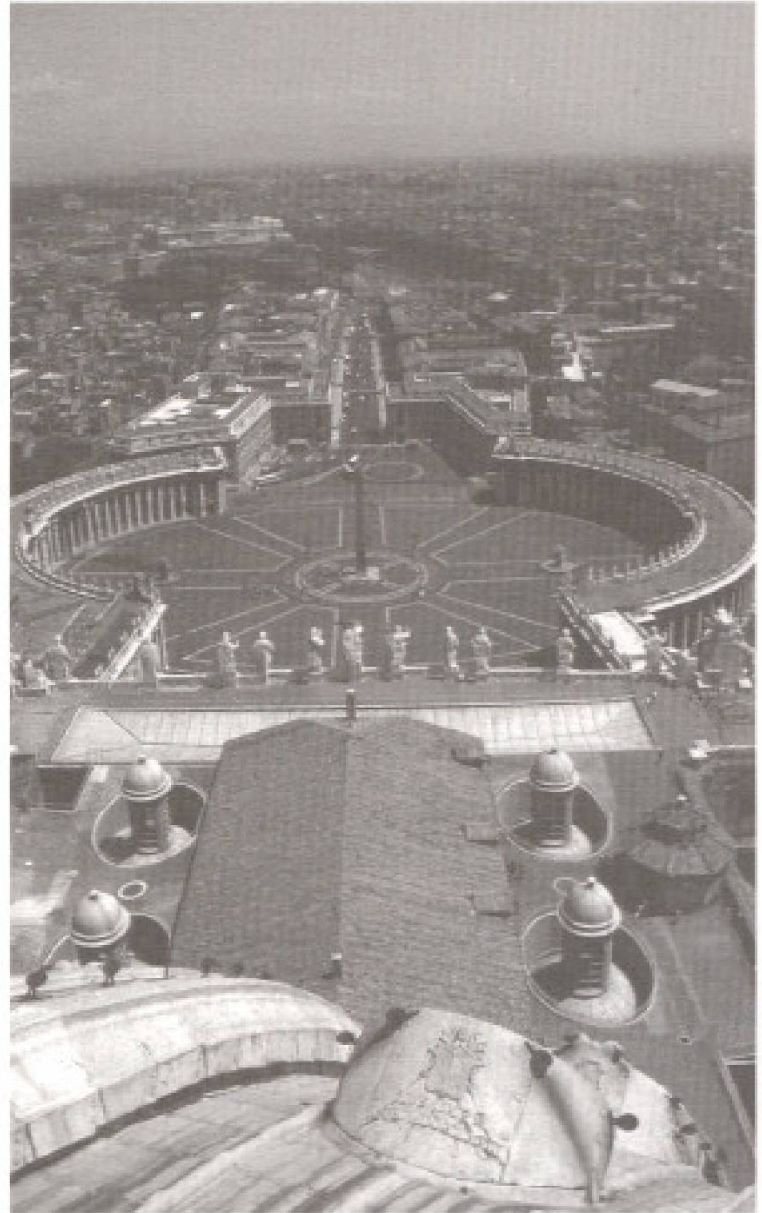

Place Saint-Pierre, Vatican.

(50 000 lits plus 15000 non commerciaux). Si l'on ajoute l'hospitalité occasionnelle ou celle d'amis, on peut arriver à 70000 à 80000 lits disponibles... dans I'hypothèse peu vraisemblable que toutes les capacités d'accueil puissent entrer dans un circuit informatique international centralisé... Il convient de ne pas oublier que Rome continuera à vivre de tourisme et d'affaires, si bien que la capacité hôtelière au service des pèlerins pourrait n'être que d'environ 15000 lits dans les hôtels et 5000 dans la périphérie proche.

Le Centre d'études du Touring Club (Qui Touring, 1997: 15) précise encore que la capacité réelle annuelle en 1995 a été dé 12827938 présences pour un maximum thérique de 18484061 et une base journalière respectivement de 35145 et de 50641 . À quoi on pourrait ajouter pour les pèlerins 5000 à 6000 lits supplémentaires dans les maisons religieuses.

La question cruciale est bien celle de la centralisation de l'information et de sa diffusion la plus large possible à tous les participants pour éviter les phénomènes ponctuels de surnombre et de paralysie, pour ne pas dire d'asphyxie.

Les organismes de tourisme italien s'emploient à utiliser l'événement pour faire valoir les immenses richesses artistiques du \& Beau Pays $*$ et attirer les visiteurs sur des circuits qui, s'ils n'évitent pas Rome, souhaitent le maximum de présences.

\section{LE SAINT-SIËGE ET LE TOURISME}

C'est le pape Paul VI qui a signé le 30 avrill 1969 le premier Directoire général pour la Pastorale du Tourisme, confiant le soin du suivi à ce qui est aujourd'hui le Conseil Pontifical pour la Pastorale des Migrants et des Itinérants. Un Observateur Permanent du Saint-Siège est nommé à l'Organisation mondiale du tourisme.

En principe, dans chaque Conférence épiscopale, un évêque préside une commission de pastorale du tourisme, du temps libre, des loisirs. Ce n'est pas encore le cas dans tous les pays, meme ceux concernés massivement par le phénomène touristique, soit comme pays émetteur, soit comme pays récepteur.

Loin d'être considéré comme un danger pour la foi ou les mours, le tourisme est vu comme une chance pour l'épanouissement de l'homme, pour la compréhension entre les cultures et les traditions si, du moins, il a visage humain. Les points centraux de la réflexion de l'Église portent sur les relations entre tourisme et développement économique et humain harmonieux, spécialement des pays récepteurs et entre tourisme et culture. Le domaine de la gestion de la Création n'échappe pas à l'attention, les risques de destruction de la nature étant dénoncés par les spécialistes internationaux comme une conséquence trop fréquente d'une expansion trop rapide et mal contrôlée des aménagements touristiques, spécialement sur le littoral et en montagne.
La clé de l'attitude pastorale de l'Église en cette matière demeure, une fois de plus, le développement intégral de l'homme, de tout l'homme et de tous les hommes.

\section{NOTE}

I P. Claudel, I'Annonce faite a Marie. Acte 1. scène 1 .

\section{BIBLIOGRAPHIE}

(1994), Tertio Millennio Adveniente, Tipografia Poliglotta Vaticana.

2000 ans de christianisme Encyclopédie.

Chelini, Jean, et Henri Branthomme (1982), Les Chemins de Dieu, Paris, Hachente.

Claudel, Paul (1938), LAnnonce faite à Marie, Acte 1, scènel.

Conseil Pontifical des Migrants, Actes des quatre Congrès mondiaux de pastorale du tourisme.

Dictionnaire d'Anheologie Chretienne.

Jean Paul II (1979). Redemplor Hominis. Tipografia Poliglotta Vaticana

Patrologie latine.

Paul VI (1969), Directoire général pour la Pastorale du tourisme.

Pietri, Charles (1976), Roma Christiana, Eoole française de Rome. 